\title{
Genetic diversity in wild Dipsacus chinensis populations from China based on ISSR markers
}

\author{
D.-X. Chen ${ }^{1,2,3}$, L.-Y. Li ${ }^{2,3}$, X. Zhang ${ }^{2,3}$, Y. Wang ${ }^{2,3}$ and Z. Zhang ${ }^{1}$ \\ ${ }^{1}$ Institute of Agricultural and Life Sciences, Chongqing University, \\ Chongqing, China \\ ${ }^{2}$ Institute of Material Medical Planting, \\ Chongqing Academy of Chinese Materia Medica, Chongqing, China \\ ${ }^{3}$ Chongqing Engineering Research Center for Fine Variety Breeding Techniques \\ of Chinese Materia Medica, Chongqing, China \\ Corresponding author: Z. Zhang \\ E-mail: zezhang@cqu.edu.cn
}

Genet. Mol. Res. 12 (2): 1205-1213 (2013)

Received July 30, 2012

Accepted October 16, 2012

Published April 12, 2013

DOI http://dx.doi.org/10.4238/2013.April.12.7

\begin{abstract}
Wild Dipsacus chinensis plants in China have become endangered owing to over-harvesting and habitat fragmentation. We examined the genetic diversity and genetic structure of 90 individuals from three populations using inter-simple sequence repeat markers and found that 106 of 173 bands amplified by 22 informative and reliable primers were polymorphic. These findings correspond to a medium level of genetic diversity. At the species level, the estimates of parameters of genetic diversity were as follows: polymorphic loci $(61.27 \%)$; effective number of alleles (1.3873); Nei's genetic diversity (0.2202); Shannon's information index (0.3235). At the population level, the estimates were polymorphic loci $(9.53 \%)$; effective number of alleles (1.0419); Nei's genetic diversity (0.0258); Shannon's information index (0.0402). Nei's coefficient of genetic differentiation was 0.8829 , which is consistent with Shannon's coefficient of genetic differentiation (0.8757). Most of the genetic variation existed among populations, and some differentiation may
\end{abstract}


have resulted from habitat fragmentation and barriers to gene flow (gene flow $=0.0663$ ). Combining our results with those of on-site field investigation, we conclude that the present genetic diversity and genetic structure of natural populations of $D$. chinensis have been strongly affected by harvesting and habitat fragmentation. We also propose strategies for the conservation of this plant.

Key words: Dipsacus chinensis; Inter-simple sequence repeat; Genetic diversity

\section{INTRODUCTION}

Dipsacus chinensis, also known as Zhonghua Xuduan and Dahua Xuduan, is a plant in the Dipsacaceae family (Institutum Botanicum Beijingense Academiae Sinicae Edita, 1975; Editorial Committee of Flora of China \& Chinese Academy of Sciences, 1986). The dried root of the plant has medicinal properties, nourishing the liver and kidneys, increasing blood flow, and improving healing of bone fractures. It has also been used to treat lower back pain, limb weakness, nocturnal emission, uterine bleeding, miscarriage, traumatic injury, metal-inflicted wounds, and several other ailments (Chen and Ai, 1997). In China, this plant is mainly distributed in Sichuan, Yunnan, and Xizhang Provinces, and it grows in shrub, grassland, and the edges of forests, streets, gutters, and flooded lands at altitudes of $2800-4000 \mathrm{~m}$. Field investigations have shown that human activities leading to deforestation and habitat deterioration have resulted in a sharp decline in the germplasm resources of $D$. chinensis. In turn, its distribution pattern has changed, with most populations presently growing along roadsides or on farmed land in conjunction with human activities. Therefore, wild populations have become isolated, and both population and individual numbers have markedly declined. This result is consistent with the fact that natural regeneration is slow, and the species faces serious threats to its survival. Therefore, conservation genetic studies must be carried out to understand the characteristics of the various remaining populations to develop effective strategies to protect this species from extinction. Several previous studies have examined D. chinensis, but they have focused on the traditional morphological classification (Ai et al., 1990), pollen (Feng et al., 2000), chemicals, and other features. No molecular data are available for $D$. chinensis.

Inter-simple sequence repeats (ISSRs) are molecular markers with primers designed based on simple sequence repeats of eukaryotic genomes without pre-cloning and sequencing (Zietkiewicz et al., 1994). These markers have good stability and large polymorphism. In recent years, this technology has been widely used in the field of traditional Chinese medicinal plant research to investigate the genetic diversity and structure of various plants (Shen et al., 2006; Yu et al., 2008; Song et al., 2010; Zhang et al., 2010; Yang et al., 2011; Senapati et al., 2011). Therefore, to understand the natural distribution, growth conditions, and ecological and biological characteristics of $D$. chinensis, we used ISSR to survey genetic structure and intraspecific genetic polymorphism in three populations of this species. Ascertaining the level of genetic variation and degree of differentiation among $D$. chinensis populations will enhance understanding of its evolutionary history and adaptation potential and hence provide basic information and a scientific basis for designing effective protective strategies. 


\section{MATERIAL AND METHODS}

\section{Plant materials}

Three natural D. chinensis populations [labeled Yunnan Zhongdian (YN), Sichuan Xiangcheng (SX), and Sichuan Kangding (SK)] representing regional terrain, topography, and climate differences and individual differences in natural geographic distribution were selected to investigate genetic diversity and structure (Table 1). Sampling was performed in November 2010. Thirty individuals from each population were sampled. To comply with the principle of uniform distribution and random sampling, individuals from various locations and positions were sampled to cover the various distribution patterns observed within populations. Fresh young leaves collected from each sampled plant were washed, dried, and placed in a resealable bag containing silica gel to hasten drying. The altitude, longitude, latitude, and other environmental characteristics of each population were recorded (see Table 1).

Table 1. Characteristics of samples used in this study.
\begin{tabular}{llccc}
\hline Population code & Geographic region & Altitude & Longitude & Latitude \\
\hline YN & Yunnan Zhongdian & 3270 & $099^{\circ} 40^{\prime} 184^{\prime \prime}$ & $27^{\circ} 52^{\prime} 633^{\prime \prime}$ \\
SX & Sichuan Xiangcheng & 3410 & $099^{\circ} 38^{\prime} 563^{\prime \prime}$ & $29^{\circ} 06^{\prime} 395^{\prime \prime}$ \\
SK & Sichuan Kangding & 3480 & $101^{\circ} 34^{\prime} 491^{\prime \prime}$ & $30^{\circ} 03^{\prime} 563^{\prime \prime}$ \\
\hline
\end{tabular}

\section{Genomic DNA extraction and detection}

A Tiangen genomic DNA extraction kit (Beijing, China) was used to isolate genomic DNA from each sample. The integrity of the DNA was detected using $1 \%$ agarose gel electrophoresis, and its concentration and purity were measured under ultraviolet light.

\section{ISSR-polymerase chain reaction (PCR) amplification and detection}

ISSR primers were synthesized by the Shanghai Sangon Biological Engineering Co., Ltd. (Shanghai, China), using sequences published by the University of British Columbia (Canada). Forty ISSR primers were screened in two randomly selected DNA templates from each population. Ultimately, 16 primers with clear and reproducible amplification products that showed relatively high polymorphism were used for formal amplification. The denaturation temperature varied between $1^{\circ}$ and $3^{\circ} \mathrm{C}$ according to the melting temperature of each primer. Primers, their sequences and annealing temperatures are shown in Table 2.

The ISSR reaction cocktail was designed as referenced in the literature (Chen et al., 2006). Briefly, a total volume of $25 \mu \mathrm{L}$ contained $1 \mathrm{X}$ PCR buffer, $1.5 \mathrm{mM} \mathrm{Mg} \mathrm{Mg}^{2+}, 200 \mu \mathrm{L}$ deoxyribonucleotide triphosphate, $0.4 \mu \mathrm{L}$ primers, $40 \mathrm{ng}$ genomic DNA, and $1 \mathrm{U}$ Taq DNA polymerase. The amplification program was set for $94^{\circ} \mathrm{C}$ for $5 \mathrm{~min}$, and then 35 cycles of denaturation at $94^{\circ} \mathrm{C}$ for $30 \mathrm{~s}$, annealing at $53^{\circ}-57^{\circ} \mathrm{C}$ for $1 \mathrm{~min}$, and extension for $1.5 \mathrm{~min}$, and a final cycle at $72^{\circ} \mathrm{C}$ for $7 \mathrm{~min}$. The products were stored at $4{ }^{\circ} \mathrm{C}$. All reactions were carried out on an $\mathrm{S} 1000^{\mathrm{TM}}$ Thermal Cycler (Bio-Rad, USA).

Using a $1 \mathrm{X}$ Tris-acetate-ethylenediaminetetraacetic acid buffer system, the amplified products were stained and separated using $1.5 \%$ agarose gel electrophoresis $(150 \mathrm{~V})$. When 
the bromophenol blue indicator was 2-3 cm from the edge of the gel, electrophoresis was stopped. The gel products were visualized and recorded using an automated imaging system (Gel Doc XR; Bio-Rad).

\section{Statistical analysis}

Only gel electrophoresis bands that were clear, stable, and easy to identify were used for statistical analysis. The electrophoresis results were translated into a binary data matrix according to presence (1) or absence ( 0 ) of bands.

Assuming that the populations used in this study were in Hardy-Weinberg equilibrium, we analyzed the results of the amplification for genetic parameters using the POPGENE 1.31 software. These parameters included the percentage of polymorphic loci (PPB), Shannon's information index (observed heterozygosity; $H_{\mathrm{SP}}$ at the species level, $H_{\mathrm{POP}}$ at the population level), Nei's genetic diversity $(H)$, observed number of alleles, effective number of alleles $\left(N_{\mathrm{E}}\right)$, total gene diversity $\left(H_{\mathrm{T}}\right)$, gene diversity within populations $\left(H_{\mathrm{S}}\right)$, genetic differentiation coefficient $\left(G_{\mathrm{ST}}\right)$, gene flow $\left(N_{\mathrm{m}}\right)$, and Nei's genetic distance $(D)$, and genetic identity $(I)$. A dendrogram was generated using unweighted pair group method with arithmetic mean clustering to analyze the genetic relationships among groups. We estimated the genetic variation among populations using Shannon's differentiation factor $\left[\left(H_{\mathrm{SP}}-H_{\mathrm{POP}}\right) / H_{\mathrm{SP}}\right]$.

\section{RESULTS}

\section{Genetic diversity in three populations of $D$. chinensis}

Evaluation of the genetic differences in three geographical populations of $D$. chinensis yielded ISSR polymorphic bands for 90 individuals by using the selected primers defined above. Overall, 22 of the 40 ISSR primers amplified 173 bands, 106 of which were polymorphic (see Table 2). Therefore, each primer yielded 5-13 bands, averaging 7.9 and 4.8 polymorphic bands per primer.

\begin{tabular}{|c|c|c|c|c|c|c|c|c|c|}
\hline Primers & Sequence & $\begin{array}{c}\text { Annealing } \\
\text { temperature }\left({ }^{\circ} \mathrm{C}\right)\end{array}$ & $\begin{array}{c}\text { Total } \\
\text { bands }\end{array}$ & $\begin{array}{l}\text { Polymorphic } \\
\text { bands }\end{array}$ & Primers & Sequence & $\begin{array}{c}\text { Annealing } \\
\text { temperature }\left({ }^{\circ} \mathrm{C}\right)\end{array}$ & $\begin{array}{l}\text { Total } \\
\text { bands }\end{array}$ & $\begin{array}{l}\text { Polymorphic } \\
\text { bands }\end{array}$ \\
\hline UBC809 & $(\mathrm{AG})_{8} \mathrm{G}$ & 55 & 8 & 5 & UBC841 & $(\mathrm{GA})_{8} \mathrm{YC}$ & 53 & 7 & 2 \\
\hline UBC811 & $(\mathrm{GA})_{8} \mathrm{C}$ & 57 & 6 & 2 & UBC844 & $(\mathrm{CT})_{8}^{8} \mathrm{RC}$ & 53 & 5 & 3 \\
\hline UBC812 & $(\mathrm{GA})_{8}^{8} \mathrm{~A}$ & 57 & 11 & 10 & UBC 855 & $(\mathrm{AC})_{8}^{8} \mathrm{YT}$ & 53 & 10 & 9 \\
\hline UBC815 & $(\mathrm{CT})_{8}^{8} \mathrm{G}$ & 55 & 9 & 8 & UBC856 & $(\mathrm{AC})_{8}^{8} \mathrm{YA}$ & 53 & 9 & 5 \\
\hline UBC 822 & $(\mathrm{TC})_{8}^{8} \mathrm{~A}$ & 55 & 5 & 2 & UBC857 & $(\mathrm{AC})_{8}^{8} \mathrm{YG}$ & 53 & 7 & 2 \\
\hline UBC824 & $(\mathrm{TC})_{8} \mathrm{G}$ & 53 & 6 & 5 & UBC866 & $(\mathrm{CTC})_{6}$ & 53 & 6 & 2 \\
\hline UBC 825 & $(\mathrm{AC})_{8}^{\mathrm{T}} \mathrm{T}$ & 53 & 5 & 2 & UBC 868 & (GAA) & 53 & 9 & 7 \\
\hline UBC826 & $(\mathrm{AC})_{8}^{8} \mathrm{C}$ & 53 & 11 & 10 & UBC873 & $(\mathrm{GAAC})_{4}$ & 53 & 9 & 3 \\
\hline UBC827 & $(\mathrm{AC})_{8} \mathrm{G}$ & 53 & 5 & 3 & UBC881 & $(\text { GGGTG })_{3}$ & 53 & 7 & 4 \\
\hline UBC834 & $(\mathrm{AG})_{8} \mathrm{YT}$ & 53 & 7 & 4 & UBC891 & $\mathrm{HVH}(\mathrm{TG})_{7}$ & 53.5 & 9 & 5 \\
\hline UBC835 & $(\mathrm{AG})_{8} \mathrm{YC}$ & 53 & 9 & 5 & Total & & & 173 & 106 \\
\hline UBC836 & $(\mathrm{AG})_{8}^{8} \mathrm{YA}$ & 53 & 13 & 8 & & & & & \\
\hline
\end{tabular}

$\mathrm{Y}=(\mathrm{C}, \mathrm{T}) ; \mathrm{R}=(\mathrm{A}, \mathrm{G}) ; \mathrm{H}=(\mathrm{A}, \mathrm{C}, \mathrm{T})$ (i.e., $\operatorname{not} \mathrm{G}) ; \mathrm{V}=(\mathrm{A}, \mathrm{C}, \mathrm{G})$ (i.e., not $\mathrm{T})$.

We found that genetic diversity in three populations of $D$. chinensis was moderate to high (Table 3). At the species level, PPB was $61.27 \%, N_{\mathrm{E}}$ was $1.3873, H$ was 0.2202 , and $H_{\mathrm{SP}}$ was 0.3235 
(see Table 3). Conversely, at the population level, PPB varied considerably within population (5.78$16.18 \%$ ) and averaged $9.53 \% ; N_{\mathrm{E}}$ was 1.0419 , and $H$ was 0.0258 . Considerable variation in Shannon's diversity index was also found within populations ( $\left.H_{\mathrm{POP}} ; 0.0245-0.0667\right)$, averaging 0.0402 .

Table 3. Genetic variation in three populations of Dipsacus chinensis.
\begin{tabular}{lccrcccc}
\hline Population & $\mathrm{N}$ & $\mathrm{K}$ & \multicolumn{1}{c}{$\mathrm{PPB}$} & $N_{\mathrm{A}}(\mathrm{SE})$ & $N_{\mathrm{E}}(\mathrm{SE})$ & $\mathrm{H}(\mathrm{SE})$ & $H_{\mathrm{O}}(\mathrm{SE})$ \\
\hline YN & 30 & 28 & $16.18 \%$ & $1.1618(0.3694)$ & $1.0687(0.2028)$ & $0.0426(0.1163)$ & $0.0667(0.1732)$ \\
SX & 30 & 12 & $6.94 \%$ & $1.0694(0.2548)$ & $1.0312(0.1441)$ & $0.0189(0.0820)$ & $0.0293(0.1214)$ \\
SK & 30 & 10 & $5.78 \%$ & $1.0578(0.2340)$ & $1.0257(0.1293)$ & $0.0158(0.0747)$ & $0.0245(0.1110)$ \\
Average & & 16.7 & $9.63 \%$ & $1.0963(0.2861)$ & $1.0419(0.1587)$ & $0.0258(0.0910)$ & $0.0402(0.1352)$ \\
Species level & 90 & 106 & $61.27 \%$ & $1.6127(0.4885)$ & $1.3873(0.3884)$ & $0.2202(0.2125)$ & $0.3235(0.3009)$ \\
\hline
\end{tabular}

$\mathrm{N}=$ sample sizes $; \mathrm{K}=$ polymorphic loci; $\mathrm{PPB}=$ percentage of polymorphic loci; $N_{\mathrm{A}}=$ observed number of alleles; $N_{\mathrm{E}}=$ effective number of alleles; $\mathrm{H}=$ Nei's (1973) genetic diversity; $H_{\mathrm{O}}=$ Shannon's information index (at the population level, $H_{\mathrm{pop}}$; at species level, $H_{\mathrm{sp}}$ ). Numbers in parentheses represent the standard error of the mean (SE). For populations abbreviations, see Table 1 .

Based on calculations of $H_{\mathrm{SP}}$ and $\mathrm{H}$, the genetic variation of the three populations could be ranked as $\mathrm{YN}>\mathrm{SX}>\mathrm{SK}$, with no differences in PPB values. Overall, the genetic diversity within each population was low and varied greatly, being the highest and lowest in the YN and SK populations, respectively.

\section{Genetic structure in three populations of $D$. chinensis}

Using POPGENE to calculate genetic variation, we found some genetic differentiation among populations. Under the assumption of genetic equilibrium, the $H_{\mathrm{T}}$ of the three populations was 0.2202 , with a value of 0.0258 for $H_{\mathrm{S}}$ and a value of 0.194 for gene diversity among populations $\left(H_{\mathrm{T}}-H_{\mathrm{S}}\right) . G_{\mathrm{ST}}$ is the most commonly used indicator to measure the proportion of genetic diversity among populations, with low values indicating that little variation is proportioned among populations and high values indicating that a large amount of variation is found among populations (Nei, 1973). For these populations, we calculated a $G_{\mathrm{ST}}$ of 0.8829 , meaning that $88.29 \%$ genetic variation existed among populations and $11.71 \%$ genetic variation occurred within populations. Therefore, genetic differentiation was greater among than within populations, suggesting that inter-group variation is the main source of diversity in wild populations of $D$. chinensis. Based on the results of Shannon's information indexes $\left(H_{\mathrm{SP}}\right.$ $=0.3235, H_{\mathrm{POP}}=0.0402$; see Table 3$)$, the Shannon's differentiation factor $\left[\left(H_{\mathrm{SP}}-H_{\mathrm{POP}}\right) / H_{\mathrm{SP}}\right]$ was 0.8757 - that is, $87.57 \%$ of the genetic variation occurred among populations and $12.43 \%$ occurred within populations. All of these results showed that the genetic differentiation was significant in these populations but mainly occurred among populations.

$N_{\mathrm{m}}$ is an indicator of the genetic exchange level between populations. Wright (1951) reported that an $N_{\mathrm{m}}$ value of $>1$ indicates certain gene flow between populations. The $N_{\mathrm{m}}[0.5$ $\left.\left(1-G_{\mathrm{ST}}\right) / G_{\mathrm{ST}}\right]$ value we obtained was 0.0663 , indicating a low level of gene exchange and a high degree of genetic differentiation among populations of $D$. chinensis.

\section{$I$ and cluster analysis in three populations of $D$. chinensis}

$G_{\mathrm{ST}}$ can assess only the degree of differentiation in a group, not determine the dis- 
tance of the genetic relationship between or among groups. Conversely, genetic identity (or genetic similarity coefficients) and unbiased genetic distance, can determine the degree of genetic closeness between two populations. $I$ and $D$ in three populations of $D$. chinensis were calculated using POPGENE (Table 4). $I$ ranged between 0.5731 and 0.9256 , with an average of 0.6996 . $D$ ranged between 0.0773 and 0.5566 , with an average of 0.3815 . $I$ was smallest $(0.5731)$ and $D(0.5566)$ was largest between the YN and SK populations. Conversely, $I$ was largest (0.9256) and $D(0.0773)$ was smallest between the SX and SK populations.

Table 4. The genetic diversity (above diagonal) and genetic distance (below diagonal) among populations of
Dipsacus chinensis.
\begin{tabular}{lccc}
\hline & & \\
\hline YN & YN & SX & SK \\
SX & 0.5106 & 0.6002 & 0.5731 \\
SK & 0.5566 & $* * * *$ & 0.9256 \\
\hline
\end{tabular}

For abbreviations, see Table 1.

Unweighted pair group method with arithmetic mean clustering was used to represent the genetic relationships among groups visually (Figure 1). From this depiction, we can infer that the genetic relationship was closest between SX and SK and farthest between SK and YN followed by SX and YN.

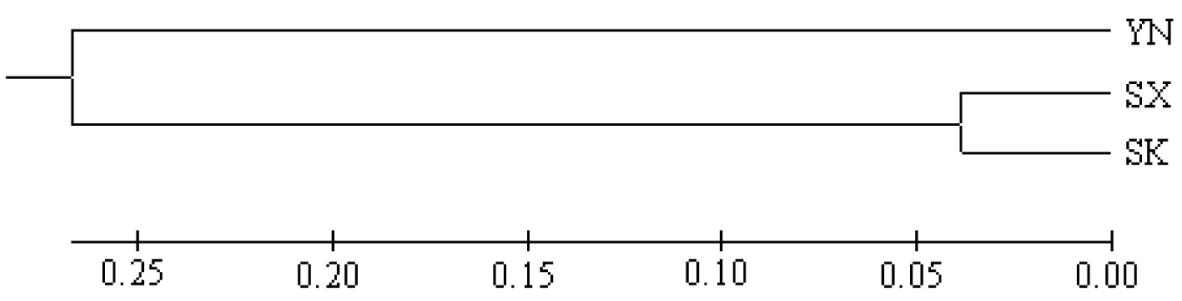

Figure 1. Dendrogram of three populations of Dipsacus chinensis. For abbreviations, see Table 1.

\section{DISCUSSION}

\section{Genetic diversity}

Genetic diversity is the core of biological diversity, reflecting the capability of a given species to adapt to the environment and thus the potential for continued evolution under environmental changes. The spatial distribution of genetic structure also reflects the evolution of ecological adaptation, environmental change, and the effects of natural selection (Solhrig, 1991). The evolutionary potential of a species and its capability to withstand adverse environmental conditions as well as the resilience and stability of its ecosystem all depend on its genetic diversity (O'Hanlon et al., 2000). Therefore, an accurate assessment of genetic variability in $D$. chinensis is a prerequisite to devising strategies for its protection.

This study revealed that genetic variation exists mainly among populations of $D$. chi- 
nensis; conversely, a narrow genetic base is present within populations. We found moderate genetic diversity at the species level (PPB $=61.27 \%$ ), but at the population level, genetic diversity was low $(\mathrm{PPB}=9.53 \%)$. We suspect the existence of an ancestor with a broad continuous distribution and rich genetic basis. However, because of human activities, habitat fragmentation has intensified, and populations with limited gene pools and discontinuous distribution have evolved. Thus, although $D$. chinensis has retained the wealth of the overall genetic diversity of its ancestor, individual numbers and population distribution have become smaller. Moreover, the small differentiation among populations and consistent genetic basis has led to relatively low genetic diversity at the population level.

\section{Genetic differentiation and $N_{\mathrm{m}}$}

The genetic structure of a plant population is affected by many factors. For instance, Hamrick and Godt (1990) showed that plant breeding systems, $N_{\mathrm{m}}$, seed dispersal mechanisms, natural selection, and other factors significantly affect the genetic structure of plants. In addition, geographic isolation can also exert some influence on genetic structure (Sagnard et al., 2002). According to our results, the main factors affecting the genetic structure of $D$. chinensis include the following: 1) geographic isolation. During the field survey, we found that the terrain distribution of $D$. chinensis was complex; hence, geographic isolation and habitat fragmentation because of mountains and canyons were obvious, hindering the flow of genes between populations. The SK and SX populations had a distribution area smaller than that of YN. In addition, population sizes differed markedly among the three populations studied. The geographic distribution characteristics showed that the distribution has obvious geographic limitations and ecological fragility. 2) Barriers to the flow of genes. In this study, $N_{\mathrm{m}}$ among populations $\left[N_{\mathrm{m}}=0.5\left(1-G_{\mathrm{ST}}\right) / G_{\mathrm{ST}}\right]$ was $<1(0.0663)$, meaning that it had been severely hampered. Moreover, this value suggested that genetic drift followed by inbreeding may have been the main impact on population genetic structure. In this regard, whereas high $N_{\mathrm{m}}$ can prevent the genetic drift caused by genetic differentiation between populations, low $N_{\mathrm{m}}$ may cause populations to adapt to the local environment, thereby promoting genetic isolation between populations ( $\mathrm{Li}$ et al., 2004).

The flow of genes among natural plant populations is mostly carried out via the spread of pollen and seeds. The pollen of $D$. chinensis is spread by insects, and therefore migrates only small distances. Hence, genes are less likely to be shared among different populations. Conversely, the spread of seeds relies on humans and animals as well as wind and gravity. Under natural conditions, gravity is the main spreading mechanism. Therefore, distance limits $N_{\mathrm{m}}$ and increased genetic differentiation among populations to some extent. Thus, it could be the main explanation for the low $N_{\mathrm{m}}$ value observed among $D$. chinensis populations. However, a full understanding of the root of limited $N_{\mathrm{m}}$ among populations of $D$. chinensis requires further exploration of the role of pollination as the mode of sexual reproduction in its natural environment.

\section{Possible causes for the endangerment in $D$. chinensis}

Public awareness of endangered plants is generally limited to the relationship between demand and supply, which ignores the possible correlation between the genetic characteristics 
of a plant population and extinction. In fact, the fate of genetics in plant populations is influenced by many factors. The data of this study could be interpreted to suggest that $D$. chinensis has high genetic diversity at the species level and hence cannot become endangered. However, on-site fieldwork has revealed the presence of numerous seedlings under mature plants. Therefore, we concluded that the seed dispersal of $D$. chinensis is weak, and because the majority of seeds rely mainly on gravity for their spread, they remained scattered near the progenitor plants. Thus, in the absence of major changes in original habitat, D. chinensis may rely on seed reproduction to maintain its populations.

Notably, we also learned through field surveys that local residents near the sampled populations had little understanding of the medicinal value of $D$. chinensis, arguing against human over-harvesting as the cause of species endangerment. However, current anthropogenic interference in the habitat of $D$. chinensis is very serious. Because its original habitat has been over-grazed or developed as farmland and seedlings have been trampled or removed as weeds, $D$. chinensis has become only sporadically distributed around the edges of alpine meadows and agricultural land or abandoned wasteland. This distribution has not only narrowed and damaged the existing habitat of $D$. chinensis but also seriously affected the probability that gravity or wind-dispersed seeds will find a suitable habitat in which to germinate. Therefore, once $D$. chinensis began to lack expansion capability, it was under threat of extinction.

In essence, $D$. chinensis became a rare species mainly owing to its special bio-ecological characteristics, which conferred poor competitiveness for survival that was further accelerated by human-induced habitat loss and consequent habitat fragmentation. Notably, a species becomes endangered as the combined result of many internal and external factors. Therefore, additional studies on the evolutionary history, breeding system, successional stage, and other inherent characteristics of $D$. chinensis are required to reveal more fully the mechanisms leading to its endangered status and thus establish reasonable and effective conservation policies and measures.

\section{Protection measures for $D$. chinensis}

Analysis of the genetic diversity of a species and the genetic structure of its population can provide important information with which to assess the protective value of rare and endangered species and develop adequate protection strategies. Our study of $D$. chinensis identified high genetic diversity at the species level, mainly among populations, and clear population differentiation. Based on these premises, we propose the following protection strategies. First, safeguard natural habitats by reducing human disturbance leading to habitat destruction and hence better protect genetic specificity. Second, protect both populations and individuals as much as possible by planning in situ conservation areas around existing natural populations, hence promoting the natural regeneration of populations. Third, manually transfer mature seeds among populations during the month of November to enhance the flow of genes. Fourth, undertake ex situ conservation strategies geared mainly toward preserving the core germplasm. Conditions permitting, sample size should be increased as often as possible. In addition, although sampling for ex situ conservation may include few samples from within populations, it should include as many as possible from different populations to improve the genetic diversity of farmed populations. 


\section{ACKNOWLEDGMENTS}

Research supported by the National Science and Technology Support Program of China (\#2006BAI06A11-09) and the Scientific and Technical Personnel Service Enterprises Action Items of the Ministry of Science and Technology in China (\#2009GJF10045).

\section{REFERENCES}

Ai TM, Chen HB, Cheng ZM and Wang YS (1990). A revision of genus Dipsacus in China. Bull. Bot. Res. 10: 1-18.

Chen DX, Li LY, Peng R and Qu XY (2006). Genetic diversity of Coptis chinensis germplasm based on ISSR analysis. Zhongguo Zhong Yao Za Zhi 31: 1937-1940.

Chen H and Ai T (1997). Medicinal plant resources of Dipsacaceae in China. Zhongguo Zhong Yao Za Zhi 22: 649-52, 702. Editorial Committee of Flora of China \& Chinese Academy of Sciences (1986). Flora Reipublicae Popularis Sinicae. Tomus 73-1. Science Press, Beijing.

Feng XF, Ai TM and Xu HN (2000). A study on pollen morphology of Dipsacus. Zhongguo Zhong Yao Za Zhi 25: 394-401. Hamrick JL and Godt MJ (1990). Allozyme Diversity in Plant Species. In: Plant Population Genetics, Breeding and Genetic Resources (Brown AHD, Clegg MT, Kahler AL and Weir BS, eds.). Sinauer Associates Inc., Sunderland, 43-63.

Institutum Botanicum Beijingense Academiae Sinicae Edita (1975). Iconographia Cormophytorum Sinicorum. Tomus IV: 340. Science Press, Beijing.

Li JM, Jin ZX and Zhong ZC (2004). RAPD analysis of genetic diversity of Sargentodoxa cuneam at different altitude and the influence of environmental factors. Acta Ecol. Sin. 24: 567-573.

Nei M (1973). Analysis of gene diversity in subdivided populations. Proc. Natl. Acad. Sci. U. S. A. 70: 3321-3323.

O'Hanlon PC, Peakall R and Briese DT (2000). A review of new PCR-based genetic markers and their utility to weed ecology. Weed Res. 40: 239-254.

Sagnard F, Barberot C and Fady D (2002). Structure of genetic diversity in Abies alba Mill. from southwestern Alps: multivariate analysis of adaptive and nonadaptive traits for conservation in France. Forest Ecol. Manag. 157: 175-189.

Senapati SK, Aparajita S and Rout GR (2011). Identification of species-diagnostic inter simple sequence repeat markers for ten Phyllanthus species. Z. Naturforsch. C. 66: 167-172.

Shen J, Ding XY, Liu DL, Ding G, et al. (2006). Intersimple sequence repeats (ISSR) molecular fingerprinting markers for authenticating populations of Dendrobium officinale Kimura et Migo. Biol. Pharm. Bull. 29: 420-422.

Solhrig OT (1991). From Genes to Ecosystems: A Research Agenda for Biodiversity. International Union of Biological Sciences, Paris.

Song Z, Li X, Wang H and Wang J (2010). Genetic diversity and population structure of Salvia miltiorrhiza Bge in China revealed by ISSR and SRAP. Genetica 138: 241-249.

Wright S (1951). The genetic structure of populations. Ann. Eugen. J. 15: 323-354.

Yang S, Chen C, Zhao Y, Xi W, et al. (2011). Association between chemical and genetic variation of wild and cultivated populations of Scrophularia ningpoensis Hemsl. Planta Med. 77: 865-871.

Yu M, Ma B, Luo X, Zheng L, et al. (2008). Molecular diversity of Auricularia polytricha revealed by inter-simple sequence repeat and sequence-related amplified polymorphism markers. Curr. Microbiol. 56: 240-245.

Zhang F, Lv Y, Dong H and Guo S (2010). Analysis of genetic stability through intersimple sequence repeats molecular markers in micropropagated plantlets of Anoectochilus formosanus Hayata, a medicinal plant. Biol. Pharm. Bull. 33: 384-388.

Zietkiewicz E, Rafalski A and Labuda D (1994). Genome fingerprinting by simple sequence repeat (SSR)-anchored polymerase chain reaction amplification. Genomics 20: 176-183. 